\title{
Developmental Pharmacology - Special Issues During Childhood and Adolescence
}

Clinical pharmacology aims to quantify drug-specific (side)-effects based on pharmacokinetics (PK) and pharmacodynamics (PD). PK (absorption, distribution, metabolism and excretion, $A D M E$ ) hereby wants to describe the drug concentration over time ('what the body does to the drug') in a specific compartment, like plasma, cerebrospinal fluid or subcutaneous tissues. PD describes the link between drug concentrations and (side)-effects over time ('what the drug does to the body'). Besides mean estimates, clinical pharmacology hereby also aims to predict and explain the extent of variability, and this is where pediatric clinical pharmacology has its specific characteristics since between and intra-patient variability is the key characteristic of children. This is because growth and development differences occur throughout the pediatric age span. These differences profoundly alter the PK and PD of drugs in children [1,2].

Weight gain, growth and maturation hereby display collinearity, but these trends are neither linear nor uniform when applied to distribution or clearance. Consequently, the key question of neonatal and pediatric pharmacotherapy is how to aim for a moving target, with an integrated appreciation on both developmental PK (ADME) and PD (effects and safety) [2,3]: preterms are not just small neonates, neonates are not just small children, and children are not small adults.

Similar to adults, the treatment of children with effective and safe medicines is crucial to improve their outcome and protect their quality of life. A rational approach to determine a safe and effective dose in an individual child necessitates understanding the PK and PD properties of a specific medicine, in combination with the clinical characteristics of a single, specific child. Modelling and simulation is one way to circumvent several difficulties in developing medicinal products in children. It allows the quantitative use of sparse sampling, characterization and prediction of PK and $\mathrm{PD}$, extrapolation from adults to children, interpolation between pediatric age subsets, optimal use of scientific literature and in vitro/preclinical data $[2,4]$. Compound specific pharmacological studies and basic developmental physiology studies describe these developmental changes and its impact on the PK/PD of drugs. Findings on specific compounds are subsequently translated to semi-physiological models that support drug PK prediction in pediatric patient cohorts [5]. However, these models need further improvement by integrating knowledge on non-maturational covariates to result in dynamic system pharmacology models [6].

The regulatory framework for pediatric medicine development in Europe hereby provides a framework on how to address pediatric drug development [4]. Authorities usually consider information regarding: (i) how similar is disease progression between adults and children, (ii) how similar is the response to intervention between these populations, and (iii) which valid and relevant PD measurements (biomarkers, outcome variables) are available to decide on the type of product development program. If it is reasonable to assume that there is a similar concentration-response in children (similar disease progression, similar response to intervention) compared to data in adults, only PK and safety studies are needed. If one does not anticipate such a similar concentration-response relationship, PK, efficacy and safety trials are needed.

Finally, besides fine tuning of drug development to pediatrics through developmental pharmacology, we should be aware that the pattern of ongoing drug evaluation studies in children almost exclusively reflects drugs initially developed for indications in adults that are subsequently considered to be adapted for the same neonatal or paediatric (e.g. infectious diseases, pain, oncology) indications [7]. However, it is obvious that pediatric product development cannot only simply copy product development from adults, but should also be driven by pediatric pathophysiology and diseases.

\section{Acknowledgements}

The clinical research of Karel Allegaert has been supported by the agency for innovation by Science and Technology in Flanders (IWT) through the SAFEPEDRUG project (IWT/SBO 130033).

\section{Conflict of Interest}

There are no conflicts of interest relevant to this paper. An annual updated version of his public declaration of interest can be retrieved on the EMA website (www.ema. europa.eu/docs/en_GB/document_library/ contacts/kallegaert_DI.pdf).

\section{Author}

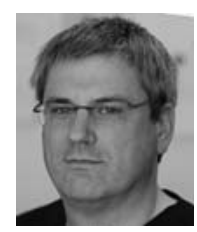

Karel Allegaert ${ }^{1,2,3}$

1 Department of Development and Regeneration, KU Leuven, Leuven, Belgium

2 Intensive Care and Department of Pediatric Surgery,

Erasmus MC-Sophia Children's Hospital, Rotterdam, Netherlands

3 Division of Neonatology, Erasmus MC-Sophia Children's Hospital, Rotterdam, Netherlands

\section{Correspondence}

\section{Karel Allegaert, MD PhD}

Department of Development and

Regeneration

KU Leuven

Herestraat 49

3000 Leuven

Belgium

karel.allegaert@uzleuven.be

k.allegaert@erasmusmc.nl

\section{References}

[1] Kearns GL, Adbel-Rahman SM, Alander SW et al. Developmental pharmacology - drug disposition, action, and therapy in infants and children. N Engl J Med 2003; 349: 11571167

[2] Brussee JM, Calvier EA, Krekels EH et al. Children in clinical trials: towards evidencebased pediatric pharmacotherapy using pharmacokinetic-pharmacodynamic modeling. Expert Rev Clin Pharmacol 2016; 9: $1235-1244$

[3] Allegaert K, van den Anker J. Neonatal drug therapy: the first frontier of therapeutics for 
children. Clin Pharmacol Ther 2015; 98 : 288-297

[4] Manolis E, Pons G. Proposals for modelbased paediatric medicinal development within the current European Union regulatory framework. Br J Clin Pharmacol 2009; 68: 493-501

[5] De Cock RF, Allegaert K, Brussee JM et al. Simultaneous pharmacokinetic modeling of gentamicin, tobramycin and vancomycin clearance from neonates to adults: towards a semi-physiological function for maturation in glomerular filtration. Pharm Res 2014; 31 : 2643-2654

[6] Allegaert K, Simons SHP, Tibboel D et al. Non-maturational covariates for dynamic systems pharmacology models in neonates, infants, and children: Filling the gaps beyond developmental pharmacology. Eur 」 Pharm Sci 2017; 109S: S27-S31

[7] Ward RM, Benjamin D, Barrett JS et al. Safety, dosing, and pharmaceutical quality for studies that evaluate medicinal products (including biological products) in neonates. Pediat Res 2017; 81: 692-711 